\title{
A two component jet model for the X-ray afterglow flat segment in short GRB 051221A
}

\author{
Z.P.Jin ${ }^{1,2}$, T. Yan $^{1,2}$, Y. Z. Fan ${ }^{1,2,3,4}$ and D. M. Wei ${ }^{1,2,5}$ \\ ${ }^{1}$ Purple Mountain Observatory, Chinese Academy of Science, Nanjing 210008, China. \\ 2 National Astronomical Observatories, Chinese Academy of Sciences, Beijing, 100012, \\ China. \\ ${ }^{3}$ The Racah Inst. of Physics, Hebrew University, Jerusalem 91904, Israel. \\ ${ }^{4}$ Golda Meir Fellow. \\ 5 Joint Center for Particle Nuclear Physics and Cosmology of Purple Mountain \\ Observatory - Nanjing University, Nanjing 210008, China.
}

\begin{abstract}
In the double neutron star merger or neutron star-black hole merger model for short GRBs, the outflow launched might be mildly magnetized and neutron rich. The magnetized neutron-rich outflow will be accelerated by the magnetic and thermal pressure and may form a two component jet finally, as suggested by Vlahakis, Peng \& Königl (2003). We show in this work that such a two component jet model could well reproduce the multi-wavelength afterglow lightcurves, in particular the X-ray flat segment, of short GRB 051221A. In this model, the central engine need not to be active much longer than the prompt $\gamma$-ray emission.
\end{abstract}

Subject headings: gamma-rays: bursts - ISM: jets and outflows - binaries: close - stars: neutron

\section{Introduction}

Kouveliotou et al. (1993) showed that the Gamma-ray Bursts (GRBs) can be divided into two distinguished groups: long GRBs (LGRBs) with duration $\geq 2$ sec and short GRBs (SGRBs) with duration $<2$ sec. Fruitful afterglow data have been collected for LGRBs and support the collapsar model (see Woosley \& Bloom 2006 for a recent review). The afterglow data of SGRBs are relatively rare and have not been reliably detected before May 2005 (Gehrels et al. 2005). The leading model for SGRBs is the merger of neutron stars or neutron star-black hole binaries (Eichler et al. 1989; Narayan et al. 1992). One attractive feature of such a compact star binaries merger model is that the accretion disk formed is 
too small to give rise to a long burst, provided that the central remnant has collapsed to a black hole immediately (e.g., Narayan et al. 2001: Rosswog et al. 2002; Aloy et al. 2005; cf. Kluźniak \& Ruderman 1998; Rosswog et al. 2003). Recently, this view of SGRB origin is supported by observations (see Mészáros 2006 for a recent review): a) For some short bursts, the host is early type galaxy, with a stellar population older than $\sim 1$ Gyr. The overall star formation rate is very low (Fox et al. 2005; Barthelmy et al. 2005). b) Deep search of an underlying supernova has resulted in null results (e.g. Hjorth et al. 2005). c) The typical number density of the medium surrounding the SGRB progenitors is low to $10^{-3} \mathrm{~cm}^{-3}$ and a stellar wind profile is disfavored.

While the compact objects binary merger model for SGRBs is largely consistent with the ongoing afterglow observations, some lightcurve data, for example, the optical or X-ray flares in GRB050709 (Fox et al. 2005) and GRB050724 (Barthelmy et al. 2005), and the X-ray afterglow flat segment in GRB051221A (Soderberg et al. 2006; Burrows et al. 2006), have not been well interpreted. One speculation is that the central engine still works after the cease of the prompt $\gamma$-ray emission (Fan et al. 2005; Zhang et al. 2006; Dai et al. 2006; Gao \& Fan 2006; Fan \& Xu 2006) and may involve some magnetic processes. For example, Fan \& Xu (2006) suggested a magnetar wind energy injection model for the X-ray afterglow flat segment lasting $\sim 10^{4}$ seconds in GRB 051221A. However, it is still unclear that whether the supermassive or hypermassive magnetar formed in the double neutron star coalescence (Price \& Rosswog 2006) could survive for such a long time (Duez et al. 2006). In this Letter we suggest a two component jet model to fit the multi-wavelength afterglow data of GRB 051221A. In this model, the central engine need not to be active much longer than the prompt $\gamma$-ray emission. We discuss the possible physical scenario giving rise to such a two component jet in section 2. We fit to the afterglow data numerically in section 3 . We summarize our result with some discussions in section 4.

\section{A two component jet model}

In the double neutron stars or neutron star-black hole merger scenario, the outflow is very likely to be neutron rich and might also be magnetized (Price \& Rosswog 2006). For a magnetized neutron-rich outflow, the acceleration process is significantly different from that of the standard fireball (Piran et al. 1993). As shown in Vlahakis, Peng \& Königl (2003), the neutron-to-proton ratio in disk-fed outflows might be as high as $\sim 30$ and the neutrons can decouple at a Lorentz factor $\Gamma \sim$ tens, while the protons continue to be accelerated to a $\Gamma \sim$ hundreds and is collimated to a narrower angle by the electromagnetic force. As a result, a two component jet might be formed(Vlahakis et al. 2003; Peng et al. 2005). We 
use such a structured jet profile to fit the afterglow of short GRB051221A in next section.

\section{Fit to the afterglow light curve of GRB 051221A}

\subsection{The observation}

GRB051221A located by Swift is one of the few SGRBs whose afterglows were well detected in multi-bands. Its duration is $T_{90}=1.4 \pm 0.2 \mathrm{~s}$ (Cummings et al. 2005), the peak energy is $E_{\mathrm{p}}=402_{-72}^{+93} \mathrm{keV}$ (Burrows et al. 2005), the gamma-ray (20keV-2MeV) fluence is about $3.2 \times 10^{-6} \mathrm{erg} \cdot \mathrm{cm}^{-2}$ (Golenetskii et al. 2005). With the optical spectrometric measured redshift $z=0.5464$ (Soderberg et al. 2006), the total isotropic gamma-ray (20keV$2 \mathrm{MeV}$ ) energy of GRB051221A is about $2.4 \times 10^{51} \mathrm{erg}$, make it the most fluent SGRB and about 6 - 35 times higher than the others (Soderberg et al. 2006). The Swift XRT began observing GRB051221A 88 seconds after the burst and found the bright, rapidly fading X-ray afterglow (Burrows et al. 2005). The X-ray afterglow early decay index is $\alpha_{\mathrm{X} 1}=1.16_{-0.17}^{+0.09}$ $(100-2200 \mathrm{~s})$ and late $\alpha_{\mathrm{X} 3}=1.09_{-0.09}^{+0.10}\left(3 \times 10^{4}-3.6 \times 10^{5} \mathrm{~s}\right)$, between them is a slow decay decline $\alpha_{\mathrm{X} 2}=0.04_{-0.21}^{+0.27}$, and the average photon spectral index of these three segments is $\Gamma_{\mathrm{X}}=2.1 \pm 0.2$ (Burrows et al. 2006). The Chandra observations show a steep decay $\alpha_{\mathrm{X} 4}=1.93_{-0.19}^{+0.23}$ after $\sim 3.6 \times 10^{5} \mathrm{~s}$, and the average photon spectral index of this segment is $\Gamma_{\mathrm{X}}=1.94_{-0.19}^{+0.29}$ (Burrows et al. 2006). The GMOS start optical observation in $r^{\prime}$ band 2.8 hours after burst and get an optical decline $\alpha_{\mathrm{opt}}=0.92 \pm 0.04$ (Soderberg et al. 2006).

The spectral analysis of the optical-to-X-ray afterglow data at $\sim 1$ day after the trigger of the burst suggests a cooling frequency $\nu_{\mathrm{c}} \sim 2 \pm 1 \times 10^{17} \mathrm{~Hz}$ (Soderberg et al. 2006). With the early X-ray decay index (100-2200s), we have an electron index $p=1-4 \alpha_{\mathrm{X} 1} / 3=$ $2.55_{-0.23}^{+0.12}$, note that $\nu_{\mathrm{c}} \propto t^{-1 / 2}$ is over the XRT band. And later $\left(3 \times 10^{4}-3.6 \times 10^{5} \mathrm{~s}\right)$, when $\nu_{\mathrm{c}}$ is crossing the X-ray band, the decay index smoothly changes from $(3-3 p) / 4$ to $(2-3 p) / 4$. The average decay index $\alpha_{\mathrm{X} 3}$ is between these two values, that we have $2.12_{-0.12}^{+0.13}=\left(2-4 \alpha_{\mathrm{X} 3}\right) / 3 \leq p \leq 1-4 \alpha_{\mathrm{X} 3} / 3=2.45_{-0.12}^{+0.13}$. Or by the optical decay index at the same time, we have $p=1-4 \alpha_{\text {opt }} / 3=2.23 \pm 0.05$ ( $\nu_{\mathrm{c}}$ is still above the optical band now). It is interesting to note that the early and later electron distribution indexes $p$ seem to be much different (see also Burrows et al. 2006 for a similar argument). In previous numerical fit to the afterglow data of GRB 051221A, a constant $p$ can not well reproduce both the early and the late X-ray declines, as shown in Figure 3 of Burrows et al. (2006) and Figure 1 of Fan \& Xu (2006). In our two component jet model, the shock parameters $\left(\epsilon_{e}, \epsilon_{B}, p\right)$ might be different for the narrow and the wide components. The early afterglow emission is mainly contributed by the narrow jet component while the late afterglow is dominated by the forward shock emission of the wide jet component. So the values of $p$ for the early and 
later afterglow data could be different.

\subsection{Numerical method}

We interpret the apparent flattening in the X-ray light curve being caused by the emergence of the forward shock emission of the wide jet component. The code here to fit the multi-band lightcurves has been used in Yan et al. (2007), in which the outflow dynamical evolution is governed by a set of differential equations.

Reverse shock phase. In this case, the energy conservation of the whole system that contains four regions (1) the unshocked medium, (2) the shocked medium, (3) the shocked ejecta, and (4) the unshocked ejecta (reads Huang et al. 2000; Yan et al. 2007)

$$
d\left(E_{2}+E_{3}+E_{4}\right)=-\varepsilon_{2} \gamma_{2} d U_{2}-\varepsilon_{3} \gamma_{3} d U_{3}
$$

where $E_{\mathrm{i}}$ is kinetic energy measured in observer frame and they are governed by $E_{2}=$ $\left(\gamma_{2}-1\right) m_{2} c^{2}+\left(1-\varepsilon_{2}\right) \gamma_{2} U_{2}, E_{3}=\left(\gamma_{3}-1\right) m_{3} c^{2}+\left(1-\varepsilon_{3}\right) \gamma_{3} U_{3}$ and $E_{4}=(\eta-1)\left(m_{\mathrm{ej}}-m_{3}\right) c^{2}$. Where $U_{2}=\left(\gamma_{2}-1\right) m_{2} c^{2}$ and $U_{3}=\left(\gamma_{34}-1\right) m_{3} c^{2}$ are internal energy measured in the comoving frame. Note that in this subsection, the subscripts $i=1-4$ represent the $i$-th region, respectively. $\varepsilon_{\mathrm{i}}$ is the radiation fraction of internal energy, $\beta_{\mathrm{i}}, \gamma_{\mathrm{i}}$ are the velocities (in units of $c$ ) and the corresponding Lorentz factors measured in observer frame, respectively; $m_{\mathrm{i}}$ is the rest mass of the material, $\gamma_{34}$ is the Lorentz factor of region 3 relative to region 4.

The mass of the medium swept by the forward shock is

$$
d m_{2}=4 \pi R^{2} n_{1} m_{p} d R
$$

where $n_{\mathrm{i}}$ is the proton number density measured in the comoving frame, $m_{\mathrm{p}}$ is the proton's rest mass and $R$ is the radius of forward shock front to the central engine.

The mass of the medium swept by the reverse shock is

$$
d m_{3}=4 \pi R^{2} \eta n_{4} m_{p}\left(\beta_{4}-\beta_{\mathrm{RS}}\right) d R
$$

where $\eta$ is the initial Lorentz factor of outflow, and $\beta_{\mathrm{RS}} \approx\left(\gamma_{3} n_{3} \beta_{3}-\gamma_{4} n_{4} \beta_{4}\right) /\left(\gamma_{3} n_{3}-\gamma_{4} n_{4}\right)$ (Sari \& Piran 1995; Fan et al. 2004) is the velocity of reverse shock.

With eq.(1-3) and assuming that $\gamma_{2}=\gamma_{3}$, we have the following equation to describe the dynamical evolution of forward shock

$$
d \gamma_{2}=-4 \pi R^{2} \frac{\left(\gamma_{2}^{2}-1\right) n_{1} m_{p}+\left(\gamma_{2} \gamma_{34}-\eta\right) \eta n_{4} m_{p}\left(\beta_{4}-\beta_{\mathrm{RS}}\right)}{I}
$$


where $I=m_{2}+m_{3}+\left(1-\varepsilon_{2}\right)\left(2 \gamma_{2}-1\right) m_{2}+\left(1-\varepsilon_{3}\right)\left(\gamma_{34}-1\right) m_{3}+\left(1-\varepsilon_{3}\right) \gamma_{2} m_{3}\left[\eta\left(1-\beta_{2} \beta_{4}\right)-\frac{\eta \beta_{4}}{\gamma_{2}^{2} \beta_{2}}\right]$.

After the cease of the reverse shock. In this case, the dynamical evolution of the forward shock has been discussed in great detail (Huang et al. 2000). The reverse shock electrons cool adiabatically. We take the analytical approach of Sari \& Piran (1999) to calculate the emission of those electrons.

In the afterglow lightcurve calculation (see Yan et al. 2007 for details), the cooling of the shocked electrons due to both synchrotron and inverse Compton has been taken into account (Sari et al. 1998; Wei \& Lu 1998; Sari \& Esin 2001). The synchrotron radiation of the forward shock electrons on the "equal arriving surface" (on which the emission reaches us at the same time) has been integrated strictly (Huang et al. 2000).

\subsection{Fit to the afterglow data of GRB051221A}

Using the code described above, we fit the afterglow of GRB051221a. Here we consider two cases. First, we assume that the shock physical parameters of two components are the same (Berger et al. 2003; Sheth et al. 2003; Huang et al. 2004). The parameters taken into account are as follow: for the narrow component, the isotropic energy $E_{\mathrm{k}, \text { iso }}=3.2 \times 10^{51}$ ergs, the initial Lorentz factor $\eta=500$, and the half opening angle $\theta_{j}=0.03$; for the wide component, $E_{\mathrm{k}, \text { iso }}=9.5 \times 10^{51} \mathrm{ergs}, \eta=50$, and $\theta_{j}=0.1$. For both components, $\epsilon_{e}=0.3, \epsilon_{B}=0.01$, and $p=2.2$. However, from Fig.1 we note that in this case the fit is not very well, the reason is that we take the same value of $p$ for the two components (similar conclusion can be found in Burrows et al. 2006 and Fan \& Xu 2006). So we take a different value of $p=2.8$ for the narrow component and find that the numerical results can well reproduces the afterglow of GRB051221A. In this case the ratio of total energy of narrow and wide component is $1: 30$, which is very different from $3: 7$, the ratio found in Vlahakis et al.'s simulation (2003). Second, we assume that for the narrow and the wide components, the shock parameters are different. This assumption is somewhat unusual. For the pre-Swift GRBs, the best fitted micro physical parameters are different from burst to burst (Panaitescu \& Kumar 2001; Yost et al. 2003). For the Swift GRBs, the situation is less clear because the afterglows are usually too peculiar to be reasonably reproduced in the standard fireball model (e.g., Fan \& Piran 2006). Figure 2 Shows our numerical fit to the X-ray and optical afterglows with the following parameters: for the narrow component, the isotropic energy $E_{\mathrm{k}, \text { iso }}=3.2 \times 10^{52} \mathrm{ergs}$, the initial Lorentz factor $\eta=500, \theta_{j}=0.03$, $\epsilon_{e}=0.09, \epsilon_{B}=0.003$, and $p=2.8$; for the wide component, $E_{\mathrm{k}, \text { iso }}=9.5 \times 10^{51} \mathrm{ergs}, \eta=50$, $\theta_{j}=0.1, \epsilon_{e}=0.3, \epsilon_{B}=0.01$, and $p=2.2$. 


\section{Summary and Discussion}

In this work we show that the multi-band afterglows of GRB051221A could be well fitted by a two component jet model. Such a two component jet might be formed if the disc-fed outflow is neutron rich and magnetized (Vlahakis et al. 2003). Our good fit to the afterglow data in turn suggests that the initial ejecta launched in the double neutron stars merger or neutron star-black hole merger may be neutron rich and magnetized. However, we point out that any model consisting of a fast and a slower jet can also provide a good fit to the observed data for 051221A. This and some other possibilities are described below.

There are several possible ways to produce a slower jet: 1) Neutrino annihilation and magnetic field were considered as the energy source for SGRB in NS binary merger scenario (Eichler et al. 1989; Narayan et al. 1992). Maybe they both operate, but only one mechanism produced the GRB prompt emission while the other contribute only to late afterglow

as a second component (Rosswog et al. 2003; Price \& Rosswog 2006). 2)A thick disc acts as high-density wall was considered to collimate GRB outflow (Rosswog et al. 2003). It was thought if mixing instabilities at the walls spoil the jet with baryons, it would result in a non-relativistic "dirty" fireball (Rosswog et al. 2003). We consider that if the disc changes to unstable when the GRB outflow launch, the consequent outflow might be more baryon loaded and less collimated, maybe as a second component. It should be noted that the validity of these processes are quite uncertain and more detailed investigation are needed before they can be used for afterglow calculations.

We would like to point out that the two component jet model is not unique to account for the afterglow data of GRB051221A. One possibility is that the GRB outflow might have a range of bulk Lorentz factors and the inner parts moving with a bulk Lorentz factor $\sim$ tens carry most of the energy (Rees \& Mészáros 1998). As the outer faster parts got decelerated by the external medium, the inner parts would catch up with the decelerating forward shock and increase its kinetic energy. For the particular GRB 051221A, an energy-lorentz factor distribution $E(>\Gamma \sim 20) \propto \Gamma^{-4.5}$ is needed to reproduce the X-ray afterglow flat segment (Soderberg et al. 2006; Burrows et al. 2006). But the physical process pumping such kind of energy injection, in particular in the SGRB scenario, is not clear yet. The other possibility is that the central engine is active much longer than the prompt $\gamma$-ray emission phase. For example, Fan \& Xu (2006) proposed a magnetar wind energy injection model to account for the long term X-ray flat segment. However, it is still unclear that whether the supermassive or hypermassive magnetar formed in the double neutron star coalescence could survive for such a long time. In principle, there could be a method to distinguish the two component jet model from these two possibilities - In the two component jet model, the first jet break might be observed when the edge of the narrow component enters our line of sight. The lack 
of a detection of the first jet break renders the energy injection model possibly. The future fruitful muti-wavelength afterglow data may help us to pin down the underlying physical processes.

\section{Acknowledgments}

We thank the referee for useful comments which helped to improve this paper. This work is supported by the National Natural Science Foundation (grants 10225314, 10233010, 10621303 and 10673034) of China, and the National 973 Project on Fundamental Researches of China (NKBRSF G19990754). 


\section{REFERENCES}

Aloy,M.A., Janka,H.-T., \& Müller.E. 2005, A\&A, 436, 273

Barthelmy,S.D., Chincarini,G., Burrows,D.N., et al. 2005, Nature, 438, 994

Berger,E., Kulkarni,S.R., Pooley,G., et al. 2003, Nature, 426, 154

Burrows,D.N., Capalbi,M., Grupe,D., et al. 2005, GCN 4365

Burrows,D.N., Grupe,D., Capalbi,M., et al. 2006, ApJ, 653, 468

Cummings,J., Barbier,L., Barthelmy,S., et al. 2005, GCN 4365

Dai,Z.G., Wang,X.Y., Wu,X.F., \& Zhang,B. 2006, Science, 311, 1127

Duez M. D., Liu Y. T., Shapiro S. L., Shibata M., Stephens B. C. 2006, Phys. Rev. D., 73, 4015

Eichler, D., Livio, M., Piran, T., \& Schramm, D. N. 1989, Nature, 340, 126

Fan,Y.Z., \& Piran, T. 2006, MNRAS, 369, 197

Fan,Y.Z., \& Xu, D. 2006, MNRAS, 372, L19

Fan, Y. Z., Wei, D. M., \& Wang, C. F. 2004, A\&A, 424, 477

Fan, Y. Z., Zhang, B., \& Proga, D. 2005, ApJ, 635, L129

Fox,D.B., Frail,D.A., Price,P.A., et al. 2005, Nature, 437, 845

Gao,W. H., \& Fan, Y. Z. 2006, Chin. J. Astron. Astrophys., 6, 513

Gehrels, N. et al. , 2005, Nature, 437, 851

Golenetskii,S., Aptekar,R., Mazets,E., et al. 2005, GCN 4394

Hjorth, J., et al. 2005a, ApJ, 630, L117

Huang,Y.F., Gou,L.J., Dai,Z.G. \& Lu,T. 2000, ApJ, 543, 90

Huang,Y.F., Wu,X.F., Dai,Z.G., Ma,H.T., \& Lu,T. 2004, ApJ, 605, 300

Kluźniak, W., \& Ruderman, M. 1998, ApJ, 505, L113

Kouveliotou, C., Meegan, C. A., Fishman, G. J., et al. 1993, ApJ, 413, L101 
Mészáros, P. 2006, Rept.Prog.Phys., 69, 2259

Narayan, R., Paczynski, B., \& Piran, T. 1992, ApJ, 395, L83

Narayan, R., Piran, T., \& Kumar, P. 2001, ApJ, 557, 949

Panaitescu,A., \& Kumar,P. 2001, ApJ, 560, 49

Peng,F., Königl,A., \& Granot,J. 2005, ApJ, 626, 966

Piran, T., Shemi, A., \& Narayan, R. 1993, MNRAS, 263, 861

Price,D.J., \& Rosswog,S. 2006, Science, 312, 719

Rees, M. J., \& Mészáros, P. 1998, ApJ, 496, L1

Rosswog,S., \& Davies,M.B. 2002, MNRAS, 334, 481

Rosswog,S., Ramirez-Ruiz,E., \& Davies,M.B. 2003, MNRAS, 345, 1077

Sari,R., \& Esin,A.A. 2001, ApJ, 548, 787

Sari,R., \& Piran,T. 1995, ApJ, 455, L143

Sari,R., \& Piran,T. 1999, ApJ, 517, L109

Sari,R., Piran,T., \& Narayan,R. 1998, ApJ, 497, L17

Sheth,K., Frail,D.A., White,S., et al. 2003, ApJ, 595, L33

Soderberg,A.M., Berger,E., Kasliwal,M., et al. 2006, ApJ, 650, 261

Vlahakis,N., Peng,F., \& Königl,A. 2003, ApJ, 594, L23

Wei, D. M., \& Lu,T. 1998, ApJ, 505, 252

Woosley, S. E., \& Bloom, J. S. 2006, ARA\&A, 44, 507

Yan,T., Wei.D.M., \& Fan,Y.Z. 2007, ChJAA submitted

Yost,S.A., Harrison,F.A., Sari,R., \& Frail,D.A. 2003, ApJ, 597, 459

Zhang,B., Fan, Y. Z., Dyks, J., Kobayashi, S., Mészáros, P., et al. 2006, ApJ, 642, 354 


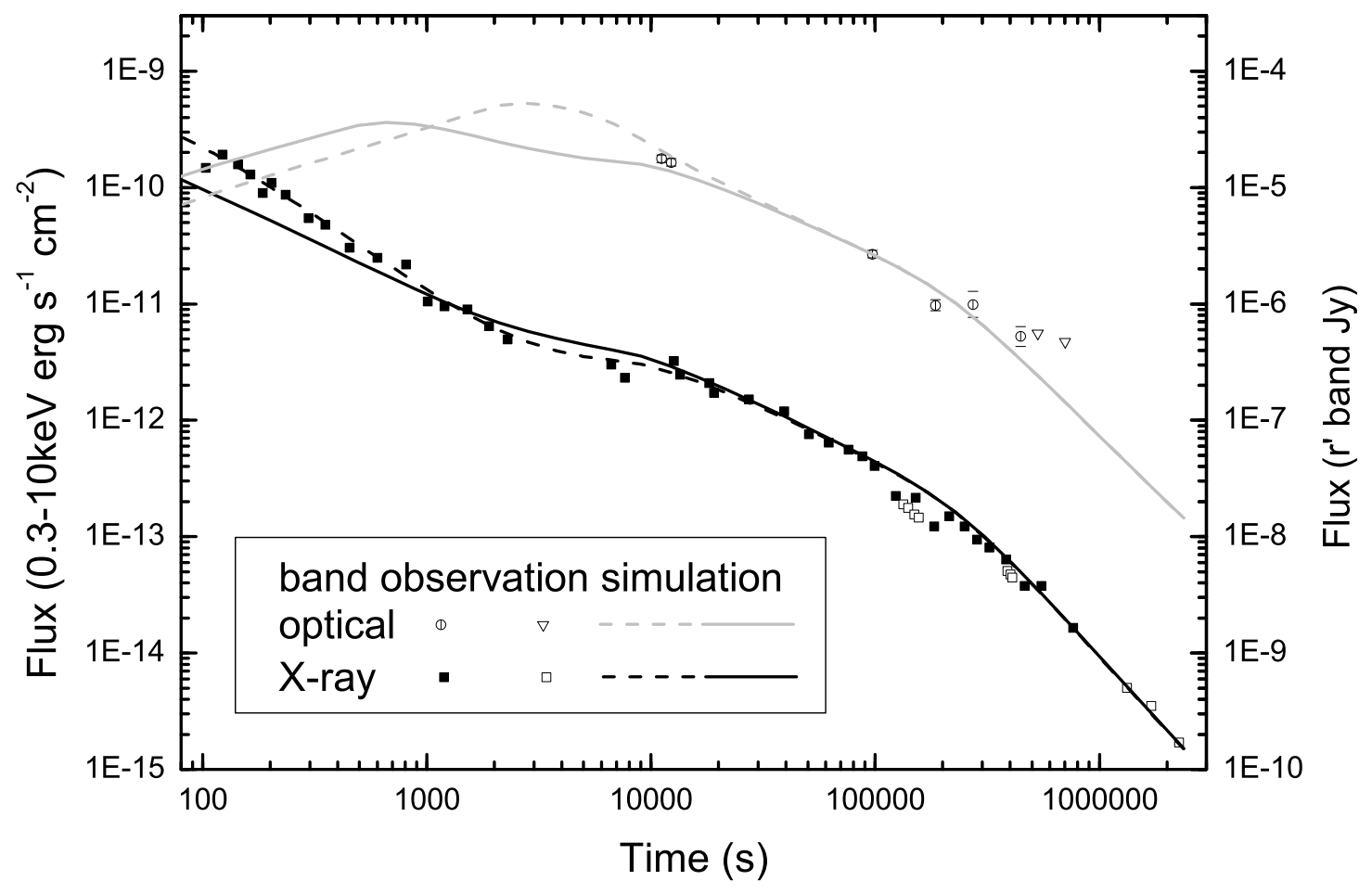

Fig. 1.- Fit to the multi-band afterglow of GRB051221A. Circles and squares are optical $\left(r^{\prime}\right.$ band) and X-ray $(0.3-10 \mathrm{keV})$ observations. The solid lines are our calculation. To make a comparison, we also take a different $p=2.8$ for the narrow jet, as the dashed lines shown. 


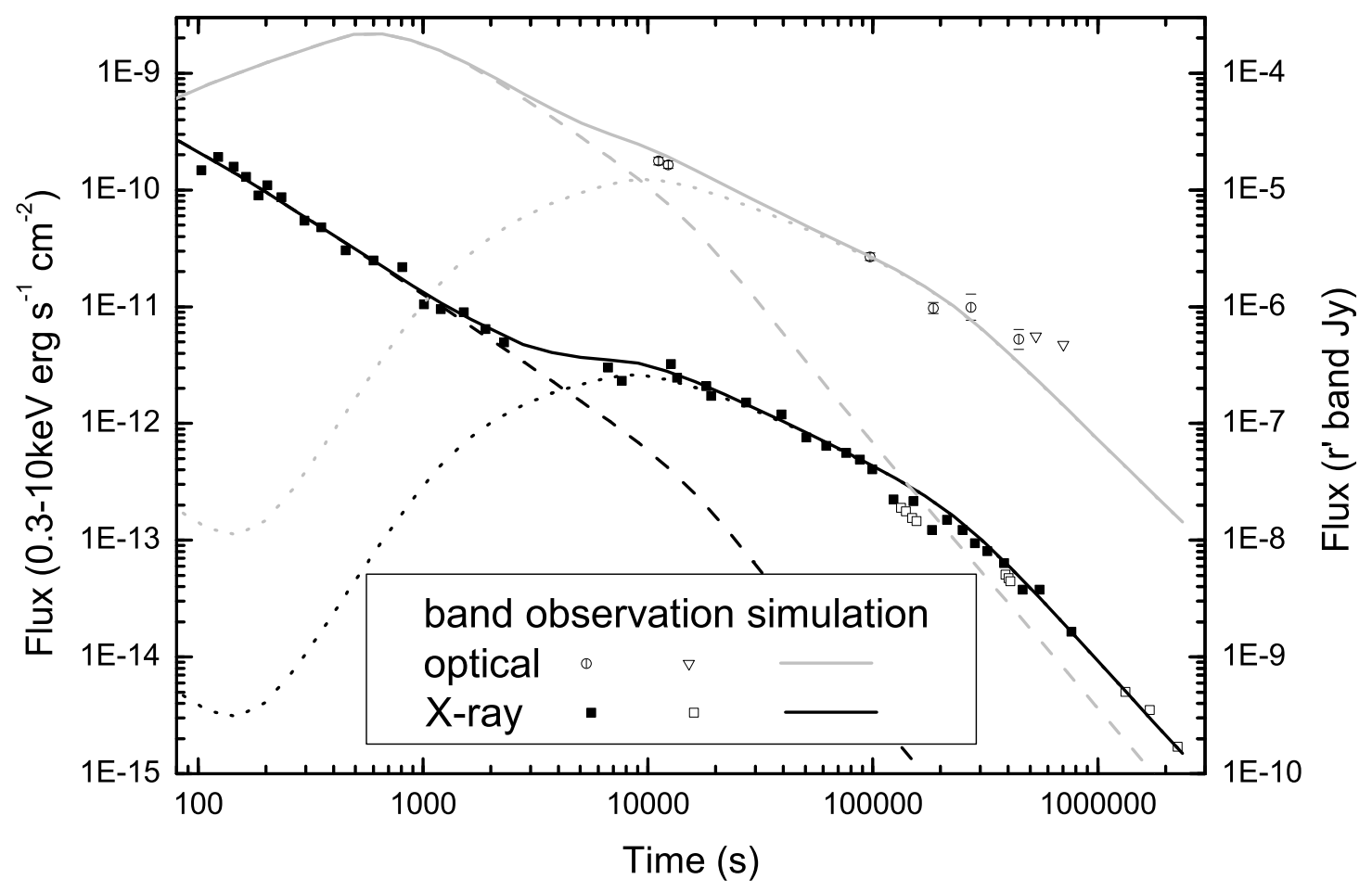

Fig. 2.- Fit to the multi-band afterglow of GRB051221A. Circles and squares are optical $\left(r^{\prime}\right.$ band) and X-ray (0.3-10keV) observations. The dashed, dotted and solid lines are narrow, wide jet and combined calculation. 